\title{
Anisotropic diffusion of spheroids in liquids: Slow orientational relaxation of the oblates
}

\author{
R. Vasanthi, Sarika Bhattacharyya, and Biman Bagchia) \\ Solid State and Structural Chemistry Unit, Indian Institute of Science, Bangalore, India 560012
}

(Received 24 September 2001; accepted 25 October 2001)

\begin{abstract}
We carry out extensive molecular dynamics (MD) simulations of translation and rotation of spheroids in the suspension of spheres, for a wide range of values of the aspect ratio, $\kappa$ ranging from the extreme limits of long rodlike molecules to the disc-shaped ones. While diffusion is anisotropic in short times (compared to the orientational correlation time), it is isotropic in the very long time and the ratio of the two components of short time translational diffusion $\left(D_{\|}\right.$and $D_{\perp}$, where $\|$ and $\perp$ refer to the motion parallel and perpendicular to the major axis) scales with the aspect ratio $\kappa$ over a large range of $\kappa$. For thin discs, the preferred mode of motion is in the plane of the disc. The oblates show a very long-time orientational correlation which is reflected by the slow decay of its time correlation function. The correlation time becomes progressively longer and finally diverges as the spherical limit is approached. This is expected because the angular momentum is conserved in the limit of fully spherical molecules $(\kappa=1)$. For both prolates and oblates, the crossover from the anisotropic to the isotropic motion is found to correlate with the orientational correlation time of the spheroid. (C) 2002 American Institute of Physics. [DOI: 10.1063/1.1428343]
\end{abstract}

\section{INTRODUCTION}

Because nonspherical molecules can rotate as it translates, the translational motion of these molecules can differ significantly from that of a sphere. ${ }^{1-3}$ The simplest example of the translation-rotation coupling is the fact that the linear momentum of a molecule can easily get converted into the angular momentum because of collision and vice versa. Thus, both the velocity and the stress time autocorrelation functions of a molecular liquid can be significantly different from that of an atomic liquid. In addition, the motion of a disclike or a rodlike molecule can be quite different from that of a spherical one and also from each other. Little is known about the shape and size dependence of diffusion. While experimental studies on diffusion almost always use nonspherical molecules, theoretical analyses of the results on spatial and orientational density relaxations in dense liquids mostly invoke spherical shape of molecules, ${ }^{4}$ with only a few notable exceptions. ${ }^{2}$ While theoretical choice of spheres is clearly motivated by the simplicity of the description ${ }^{4}$ (even hydrodynamic solutions for nonspherical molecules are not always available in closed form), it does make the analysis of the experimental results tentative. The situation is particularly anomalous when one uses a theory developed for spheres, such as the mode coupling theory in its standard form, to explain orientational relaxation, as measured by dielectric, NMR or fluorescence depolarization experiments.

The stick hydrodynamic theory predicts that for rods, the translational diffusion coefficient in the parallel $\left(D_{\|}\right)$direction (parallel to its major axis) is only twice of that in the perpendicular direction $\left(D_{\perp}\right) .{ }^{5}$ With the slip hydrodynamic boundary condition, it is shown that there is a decoupling

\footnotetext{
a) Author to whom correspondence should be addressed. Electronic mail: bbagchi@sscu.iisc.ernet.in
}

between the parallel and perpendicular motion ${ }^{6,7}$ and the ratio of the diffusion coefficients in the parallel and perpendicular direction approaches $\kappa$. Also for the rotational friction on a sphere, slip and stick hydrodynamic boundary conditions vary a lot. ${ }^{8}$

Very little is known about the anisotropic diffusion of disc-shaped molecules, i.e. of the oblates. It is expected that a disc-shaped molecule will translate preferably in the plane of the disc (saucerlike motion), although tumbling of its axis may serve to change its direction of motion. The degree of anisotropy should, like the rods, depend on the aspect ratio. Orientational relaxation (OR) of oblates is also of interest. Note that most experiments of OR (fluorescence depolarization, NMR, Kerr) measure the time correlation of the second rank spherical harmonics, $Y_{2 m}(\boldsymbol{\Omega}(t))$, where $\boldsymbol{\Omega}(t)$ is the time-dependent orientation of the oblate. For an oblate, one essentially studies $P_{2}(\cos \theta(t))$ where, $P_{2}$ is the second rank Legendre polynomial of the angle that the major axis of the oblates makes with the Z-axis of the laboratory fixed frame. Only dielectric relaxation probes the first rank, $P_{1}(\cos \theta(t))$. The time correlation function of $P_{2}$ and $P_{1}$ can be widely different - a fact not always recognized.

In a series of papers Medina et $a l^{2,9}$ investigated both translational and rotational diffusion of nonspherical molecules in suspension of spheres, by using a microscopic theoretical approach. Theoretical studies of Medina et al. have suggested a strong coupling between the rotational and the translational motions. A similar coupling was earlier obtained by Chandra and Bagchi in their study of orientational relaxation of dipolar molecules. ${ }^{10,11}$

The main motivation of this work is to understand the translational-rotational coupling of these nonspherical molecules. The diffusion of the prolates has been studied earlier $^{12}$ and here we present molecular dynamics (MD) 
simulations of tagged spheroids (both oblates and prolates) in a liquid of Lennard-Jones spheres. We find that the translational diffusion of the oblates is strongly coupled with the rotational diffusion and we compare the simulation results with the stick hydrodynamic theory. Also the ratio, $D_{\|} / D_{\perp}$, varies linearly even for the oblates similar to the prolates. The average orientational relaxation time is found to diverge as the $\kappa=1$ limit is approached, both from the oblate and prolate side. This happens because in the spherical limit the angular momentum is conserved and, thus, the rotational correlation time diverges.

The rest of the paper is organized as follows. In the next section, we discuss the system and the simulation details, followed by the hydrodynamic predictions in Sec. III, the results and discussion in Sec. IV, and we conclude with a discussion in Sec. V.

\section{SYSTEM AND SIMULATION DETAILS}

Molecular dynamics simulation has been carried out for a system of 512 particles of which 10 are Gay-Berne (GB) ellipsoids with the remaining 502 being LJ spheres. The interactions between the particles are modeled by different interaction potentials.

The interaction between the spheres is given by the Lennard-Jones potential

$$
U_{\mathrm{LJ}}=4 \epsilon_{0}\left[\left[\frac{\sigma_{0}}{r}\right]^{12}-\left[\frac{\sigma_{0}}{r}\right]^{6}\right],
$$

where $\sigma_{0}$ is the molecular diameter, $r$ is the interparticle distance, and $\epsilon_{0}$ is the energy parameter.

The ellipsoids are aspherical molecules characterized by the aspect ratio $\kappa$. Interaction among them can be modeled by the Gay-Berne potential. ${ }^{13,14}$

The GB potential provides the interaction energy between the ellipsoids with arbitrary orientations. The expression for the GB potential is given by ${ }^{13}$

$$
\begin{aligned}
U_{\mathrm{GB}}= & 4 \epsilon\left(\hat{r}, \hat{u}_{1}, \hat{u}_{2}\right)\left[\left[\frac{\sigma^{\prime}}{r-\sigma\left(\hat{r}, \hat{u}_{1}, \hat{u}_{2}\right)+\sigma^{\prime}}\right]^{12}\right. \\
& \left.-\left[\frac{\sigma^{\prime}}{r-\sigma\left(\hat{r}, \hat{u}_{1} \hat{u}_{2}\right)+\sigma^{\prime}}\right]^{6}\right],
\end{aligned}
$$

$\sigma^{\prime}$ being the diameter of the minor axis of the ellipsoid, and $\sigma\left(\hat{r}, \hat{u}_{1}, \hat{u}_{2}\right)$ is given by

$$
\begin{aligned}
\sigma\left(\hat{r}, \hat{u}_{1}, \hat{u}_{2}\right)= & \sigma^{\prime}\left[1-\frac{1}{2} \chi\left[\frac{\left(\hat{r} \cdot \hat{u}_{1}+\hat{r} \cdot \hat{u}_{2}\right)^{2}}{1+\chi\left(u_{1} \cdot u_{2}\right)}\right.\right. \\
& \left.\left.+\frac{\left(\hat{r} \cdot \hat{u}_{1}-\hat{r} \cdot \hat{u}_{2}\right)^{2}}{1+\chi\left(u_{1} \cdot u_{2}\right)}\right]\right]^{-1 / 2},
\end{aligned}
$$

with

$$
\chi=\frac{\sigma_{\|}^{2}-\sigma_{\perp}^{2}}{\sigma_{\|}^{2}+\sigma_{\perp}^{2}},
$$

for prolates and

$$
\chi=\frac{\sigma_{\perp}^{2}-\sigma_{\|}^{2}}{\sigma_{\|}^{2}+\sigma_{\perp}^{2}}
$$

for the oblates, where $\hat{u}_{1}$ is the axial vector of the molecule $i$ and $r=r_{2}-r_{1}$, and where $r_{1}$ and $r_{2}$ denote the centers of mass of molecule 1 and 2 , respectively. $\sigma\left(\hat{r}, \hat{u}_{1}, \hat{u}_{2}\right)$ and $\epsilon\left(\hat{r}, \hat{u}_{1}, \hat{u}_{2}\right)$ are the orientation-dependent range and strength parameters, respectively. $\sigma$ and $\epsilon$ depend on the aspect ratio $\kappa$.

Finally, the interaction between a sphere and an ellipsoid is accounted by the GBLJ potential as given below ${ }^{15}$

$$
U_{\mathrm{GBLJ}}=4 \epsilon_{0}\left[\left[\frac{\sigma(\theta)}{r}\right]^{12}-\left[\frac{\sigma(\theta)}{r}\right]^{6}\right],
$$

with

$$
\sigma(\theta)=\frac{(b+R)}{\sqrt{1-X^{2} \cos ^{2} \theta}} .
$$

The aspect ratio for the ellipsoid is given by $\kappa=a / b$ and $\theta$ is the angle between the vector joining the center of the sphere and the ellipsoid and $a$, the length of the ellipsoid both for the prolates and the oblates.

$$
R=\sigma_{0} / 2,
$$

where $R$ is the radius of the sphere

$$
X=\sqrt{\frac{\bar{a}^{2}-b^{2}}{\bar{a}^{2}+R^{2}}},
$$

and

$$
\bar{a}=\sqrt{\left(b^{2}+R^{2}\right)\left(\frac{a+R}{b+R}\right)^{2}-R^{2}} .
$$

The simulations have been carried out in a cubic box by imposing the periodic boundary conditions. All the quantities in the simulation are scaled to appropriate units. The reduced density, $\rho^{*}$ the temperature, $T^{*}$ and the time step, $\Delta t$ are 0.7 , 1.0 and 0.002 , respectively. Separate simulations are carried out for particles ranging from aspect ratio, 0.25 to 4 . Simulations for aspect ratio 3.5 and 4 has been carried out in a system containing $950 \mathrm{LJ}$ spheres. In this work, aspect ratio has been varied by varying the length of the major axis, $a$.

\section{HYDRODYNAMIC PREDICTIONS}

The stick hydrodynamic prediction for both the translation and rotational motion of the spheroids are given in the following.

\section{A. Translational diffusion}

The stick hydrodynamic theory predicts that for a rod, the ratio of the parallel diffusion coefficient to that of the perpendicular coefficient, is always two irrespective of the value of the aspect ratio $\kappa$ and they are given by ${ }^{5}$

$$
\begin{aligned}
& D_{\|}=\frac{k_{B} T \ln (L / b)}{2 \pi \eta L}, \\
& D_{\perp}=\frac{k_{B} T \ln (L / b)}{4 \pi \eta L},
\end{aligned}
$$

where $L$ and $b$ are the length and diameter of the rod, respectively. 
The predictions for ellipsoids are also known and they are given by ${ }^{16}$

$$
D_{\|}=\frac{\left(2 a^{2}-b^{2}\right) S-2 a}{\left(a^{2}-b^{2}\right) 16 \pi \eta}
$$

and

$$
D_{\perp}=\frac{\left(2 a^{2}-3 b^{2}\right) S+2 a}{\left(a^{2}-b^{2}\right) 32 \pi \eta},
$$

where $a$ and $b$ are the lengths of the semi-major and semiminor axes of the ellipsoid and

$$
\begin{aligned}
& S(\text { prolate })=\frac{2}{\left(a^{2}-b^{2}\right)^{1 / 2}} \log \frac{a+\left(a^{2}-b^{2}\right)^{1 / 2}}{a} \\
& S(\text { oblate })=\frac{2}{\left(a^{2}-b^{2}\right)^{1 / 2}} \tan ^{-1} \frac{\left(b^{2}-a^{2}\right)^{1 / 2}}{a} .
\end{aligned}
$$

For $\kappa>2, D_{\|} / D_{\perp}$ behaves similarly for the rods and the prolates.

\section{B. Rotational diffusion}

According to the stick hydrodynamic theory, the rotational diffusion coefficient for the oblate and the prolate ellipsoids is given by, ${ }^{3}$

$$
D_{R}=\frac{3}{2} \frac{\kappa\left[\left(2 \kappa^{2}-1\right) S-\kappa\right]}{\kappa^{4}-1} D_{s},
$$

where

$$
D_{s}=\frac{k_{B} T}{6 V \eta},
$$

with $V$ being the volume $=\frac{4}{3} \pi r^{3}, r$ is the radius of the sphere and $S$ is defined separately for the oblates and the prolates as follows:

$$
\begin{aligned}
& S(\text { oblate })=\left(1-\kappa^{2}\right)^{-1 / 2} \tan ^{-1}\left[\frac{\left(1-\kappa^{2}\right)^{1 / 2}}{\kappa}\right], \\
& S(\text { prolate })=\left(\kappa^{2}-1\right)^{-1 / 2} \ln \left[\kappa+\left(\kappa^{2}-1\right)^{1 / 2}\right] .
\end{aligned}
$$

In the model Hamiltonian used here, there is no friction on the rotational motion about its own axis. The above expression is for the tumbling motion only and rotation about the major axis has a different expression for stick boundary condition.

\section{RESULTS AND DISCUSSION}

In this section we present the simulation results for the oblates and also explore the crossover from oblates to prolates and compare with the above-mentioned hydrodynamic predictions.

In Fig. 1 we plot the mean-square displacement of an oblate (with $\kappa=0.5$ ) in the parallel and the perpendicular directions, with respect to the oblate's orientation at time $t$ $=0$. The anisotropy is observed even for a long time in case of the discs. This is because of the presence of orientational correlations even at a long time which shows a strong translation-rotation coupling. During this time, the motion of the oblate takes place mainly in the plane of the disc

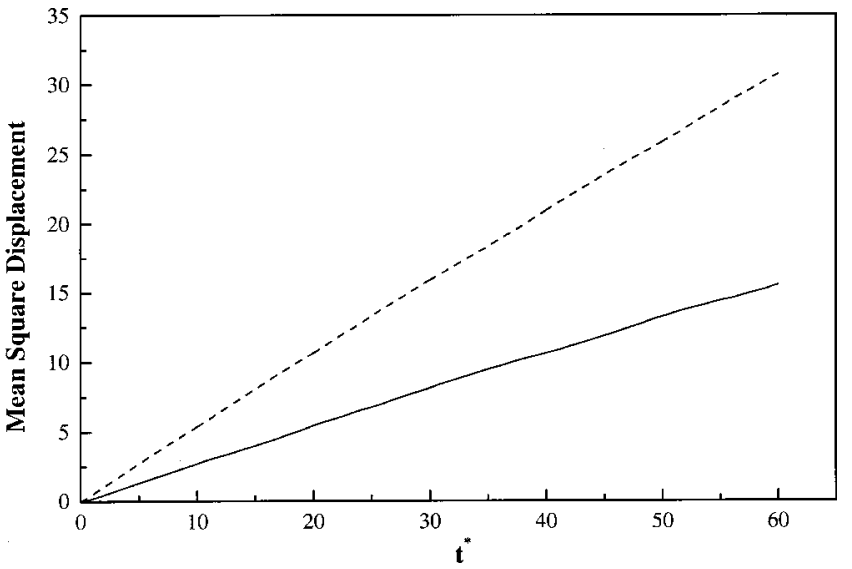

FIG. 1. The parallel and the perpendicular components of the mean-square displacement (MSD) for the oblates with $\kappa=0.5 \rho^{*}=0.7$ and $T^{*}=1.0$. The dashed line is the perpendicular component of MSD and the solid line is the parallel component of MSD.

because the motion along the plane is much faster than that along the parallel direction. A detailed analysis of the individual orientational trajectories shows that the motion of most of the oblates is wobbly and the preferred direction of motion is in the perpendicular plane. While a few of the oblates turn around fully during their motion, the majority retain their average orientation and this is discussed later on. In our earlier studies, ${ }^{12}$ we found that the prolates show anisotropy substantially for a short duration and also a shorter orientational correlations.

In Fig. 2 we plot the ratio $D_{\|} / D_{\perp}$ against the aspect ratio, $\kappa$ over a large range of $\kappa$, ranging from well below unity (the disc limit) to a substantially larger (the needle limit) limit. It is found that the ratio $D_{\|} / D_{\perp}{ }^{12,17}$ increases linearly with the aspect ratio, in agreement with the prediction of Evans et al. ${ }^{6,7}$ Note that this proportionality to the aspect ratio seems to hold even to low values like 0.3 but breaks down for aspect ratios below 0.3. Stick hydrodynamics is seen to fail, not only for prolates (rods), but also for the oblates (discs).

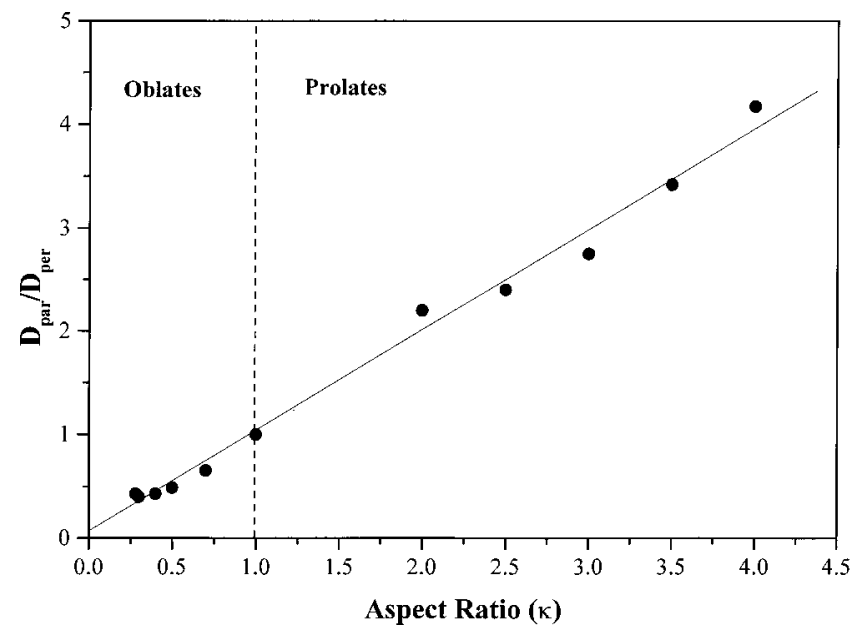

FIG. 2. The simulated ratio, $D_{\|} / D_{\perp}$ against the aspect ratio, $\kappa$ (filled circles). The solid line shows the prediction of slip hydrodynamic boundary condition. The dashed line indicates the sphere. 


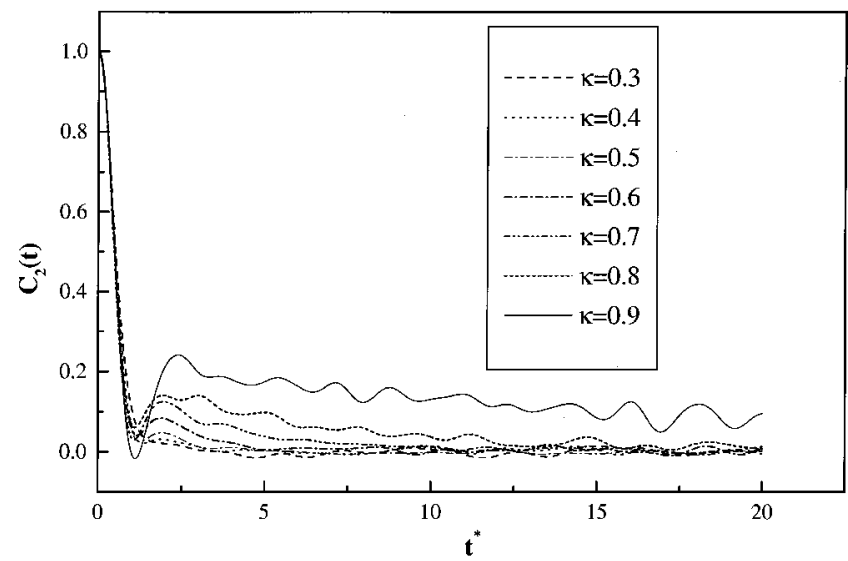

FIG. 3. The variation of second rank orientational correlation function $C_{2}(t)$ with time for various aspect ratios ranging from 0.3 to 0.9 at density $\rho^{*}=0.7$ and temperature, $T^{*}=1.0$.

In Fig. 3 we show the orientational time-correlation function (OCF) of the second rank spherical harmonics, $C_{2}(t)$, for an oblate with different aspect ratios ranging from 0.25 to 0.9 . The orientational correlation is Gaussian at short time and shows small oscillations and they persist for a very long time. The OCF of disclike oblates shows a very slow long-time decay, unlike the prolates. The reason for the slow decay of the oblates is as follows. When an oblate turns by a significant amount, it finds a barrier near $\theta=\pi / 2$ due to the nearest-neighbor solvent molecules. Thus, either it goes over the barrier and reaches $\theta=\pi$ configuration or returns to $\theta=0$. In both the cases, $P_{2}(\cos \theta(t))$ does not register any change and, therefore, does not give rise to any decay. This oscillatory mode of motion did come as a surprise to us. The study of individual orientational trajectories shows that the motion of the disc is mostly on the plane perpendicular to its major axis and tumbles about its major axis for a long time, which is reflected in the orientational correlation function, $C_{l m}(t)$. On the other hand, the prolate ellipsoids prefer to move along their major axis as their motion is hindered mostly in the perpendicular plane.

The individual orientational trajectories of the oblates has been studied and in Fig. 4 we plot the instantaneous

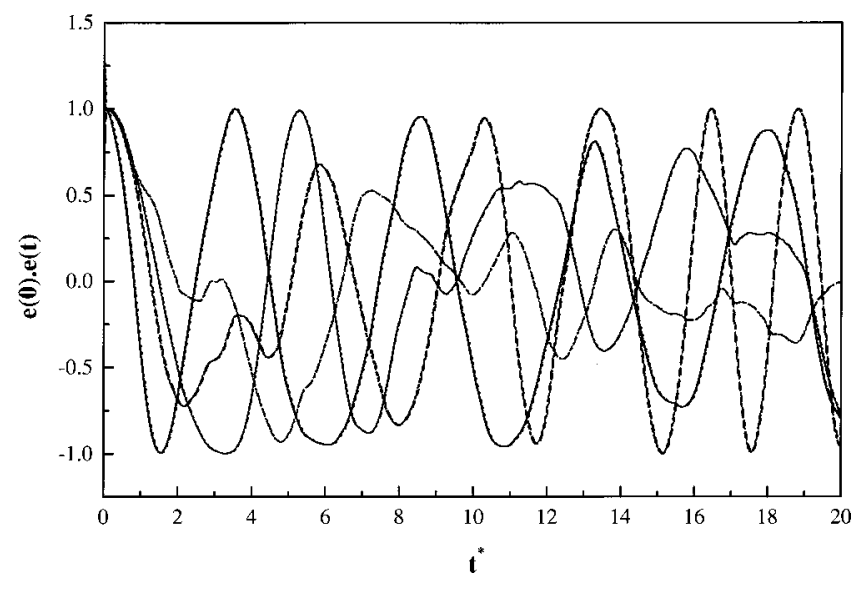

FIG. 4. Orientational trajectory $e(0) \cdot e(t)$ for all the ten oblates at reduced density, $\rho^{*}=0.7$ and temperature, $T^{*}=1.0$.

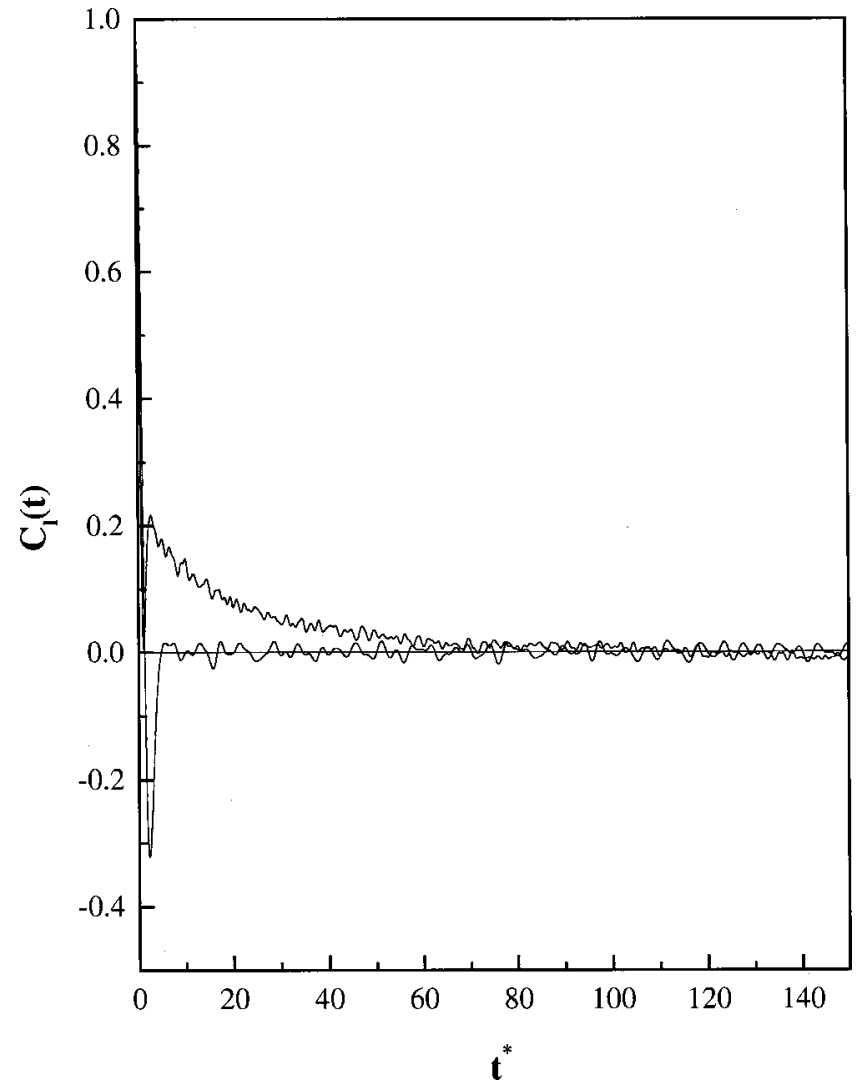

FIG. 5. Orientational correlation function of rank 1 and 2 for the oblate with aspect ratio, $\kappa=0.9$. The reduced density, $\rho^{*}=0.7$ and temperature, $T^{*}$ $=1.0$. Dashed line shows $C_{1}(t)$ and solid line shows $C_{2}(t)$.

orientational correlation $(e(t))$ with its initial orientation $(e(0)), e(0) \cdot e(t)$ against the time, for discs with aspect ratio, $\kappa=0.7$. It is observed that, while many of the discs retain their orientational correlations, a few of them decay over a long time depending on the angle by which the oblate rotates. Because of the presence of the orientational correlations, the translational motion of the oblate remains anisotropic for a long time as discussed in Fig. 1. Note that $C_{2}(t)$ does not decay when the disc rotates full circle.

In Fig. 5, we plot the orientational correlation function (OCF) of $1 \mathrm{st}\left(C_{1}(t)\right)$ and 2 nd rank $\left(C_{2}(t)\right)$ for the oblates with aspect ratio, $\kappa=0.9$ at a reduced density $\rho^{*}=0.7$ and temperature, $T^{*}=1.0$. Both $C_{1}(t)$ and $C_{2}(t)$ behave differently. While the 1 st rank OCF decays fast, the 2 nd rank OCF decays after a very long time. Small oscillations are observed in both $C_{1}(t)$ and $C_{2}(t)$. In case of the prolates, OCF of both the ranks decay in a substantially shorter duration of time.

We next present the results that show the crossover from discs to rods.

In Fig. 6 we show the variation of the product of total translational diffusion coefficient and relaxation time, $D_{T} \tau_{2 R}$ with the aspect ratio, $\kappa$ and it is compared with the stick hydrodynamic theory. ${ }^{3,16}$ It is observed that the simulated result (filled circles) does not agree at all with the stick hydrodynamic theory (solid line) and it is seen to be in error nearly by a factor of the aspect ratio, $\kappa$. Hence, the stick hydrodynamic theory prediction has been scaled by the aspect ratio, $\kappa$. The scaled hydrodynamic theory is also shown 


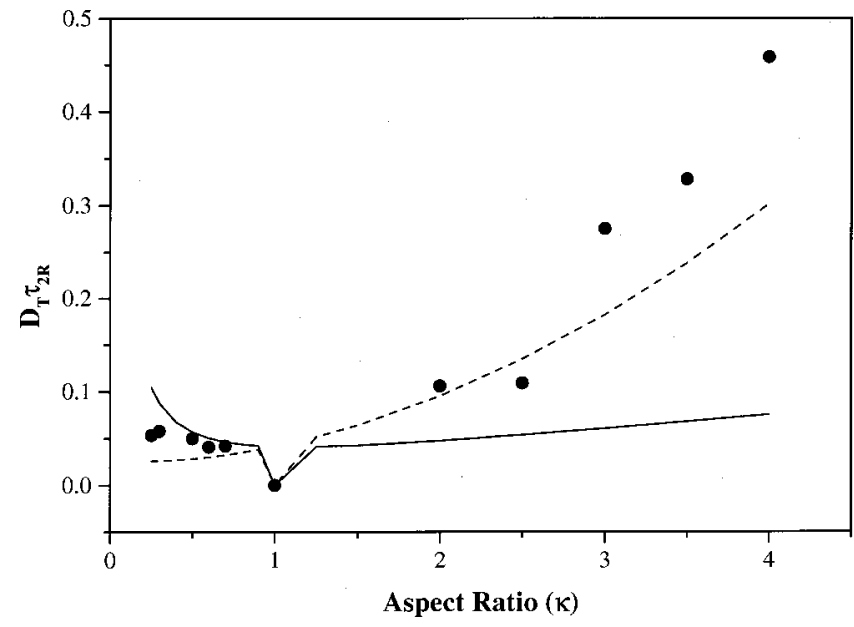

FIG. 6. Variation of the product $D_{T} \tau_{2 R}$ with aspect ratio, $\kappa$ at density, $\rho^{*}=0.7$ and temperature, $T^{*}=1.0$. The solid line shows the prediction of stick hydrodynamic theory. The dashed line shows the scaled stick hydrodynamic theory by a factor of $\kappa$. The filled circles are the simulated points.

in the figure (dashed line). After scaling, we observe that the results are qualitatively in better agreement with the theory, especially for the intermediate values of the aspect ratio, $\kappa$. According to Doi-Edwards, for the rods, the total translational diffusion coefficient varies inversely as the length of the rod, $L$ and the relaxation time, $\tau_{2 R}$ varies as $L^{3}$ and hence the product should be proportional to $L^{2}$. The simulation results for the prolate ellipsoids is in agreement with this prediction of the stick hydrodynamic theory for the rods. ${ }^{5}$ This has already been discussed before. ${ }^{2}$ However, the breakdown of the theory near $\kappa=1$ is surprising. We are not aware of any theory to explain the results.

The variation of the orientational relaxation time, $\tau_{2 R}$ with the aspect ratio, $\kappa$ ranging from 0.25 to 4 is shown in Fig. 7. In the spherical limit due to the conservation of the angular momentum the orientational relaxation time, $\tau_{2 R}$ di-

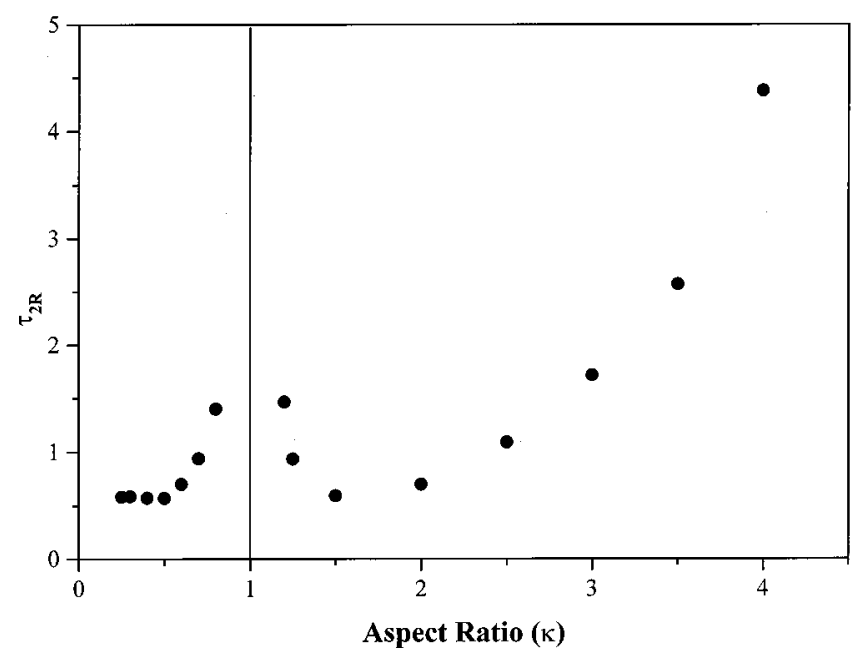

FIG. 7. Plot of the simulated orientational relaxation time, $\tau_{2 R}$ against the aspect ratio, $\kappa$. The solid line shows spherical limit. verges. The plot also shows that the orientational relaxation depends weakly on the aspect ratio, $\kappa$, for small $\kappa(\kappa<0.3)$.

\section{CONCLUSION}

The translational and rotational diffusion of tagged prolate ellipsoids has been studied in our earlier simulations. The diffusion of disc-shaped particles (oblates) are also of great interest. Here we have presented the molecular dynamics simulations of tagged ellipsoids in a sea of LJ spheres. The results here are mainly for the discs (oblates).

Both the oblates and the prolates show a linear variation of the ratio, $D_{\|} / D_{\perp}$ with the aspect ratio, $\kappa$ showing the validity of the slip hydrodynamic boundary condition. From Fig. 5, it is clear that the product $D_{T} \tau_{2 R}$ calculated from the stick hydrodynamic theory needs to be scaled nearly by a factor of $\kappa$. The most striking result of the present study is the observation of a very slow decay of the second rank orientational correlation function, $C_{2}(t)$. As discussed earlier, this slowness is due to the existence of a solvent induced barrier at midangle $(\theta=\pi / 2)$. These strong correlations are also reflected in the mean-square displacement of the oblates where the anisotropy persists for a very long time, unlike the prolate ellipsoids. As the aspect ratio approaches unity from both the sides (prolate and oblate), the orientational relaxation time, $\tau_{2 R}$, diverges.

\section{ACKNOWLEDGMENTS}

It is pleasure to thank Dr. S. Ravichandran for help and correspondence. The authors thank G. Srinivas and Arnab Mukherjee for help and discussions. This work was supported in parts by the Department of Science and Technology and the Council of Scientific and Industrial Research, India.

${ }^{1}$ B. J. Berne and R. Pecora, Dynamic Light Scattering (Wiley, New York, 1976), Chap. 7.

${ }^{2}$ M. Hernandez-Contreras and M. Medina-Noyola, Phys. Rev. E 54, 6586 (1996)

${ }^{3}$ G. R. Fleming, Chemical Applications of Ultrafast Spectroscopy (Clarendon, Oxford, 1986).

${ }^{4}$ U. Balucani and M. Zoppi, Dynamics of Liquid State (Clarendon, Oxford, 1994).

${ }^{5} \mathrm{M}$. Doi and S. F. Edwards, The Theory of Polymer Dynamics (Clarendon, Oxford, 1986), Chap. 8.

${ }^{6}$ S. Tang and G. T. Evans, Mol. Phys. 80, 1443 (1993).

${ }^{7}$ S. Tang, G. T. Evans, C. P. Mason, and M. P. Allen, J. Chem. Phys. 102, 3794 (1995).

${ }^{8}$ C. M. Hu and R. Zwanzig, J. Chem. Phys. 60, 4354 (1985).

${ }^{9}$ F. de J. Guevara-Rodriguez and M. Medina-Noyola, Phys. Rev. E 61, 6368 (2000).

${ }^{10}$ A. Chandra and B. Bagchi, J. Chem. Phys. 91, 1829 (1989).

${ }^{11}$ B. Bagchi and A. Chandra, Adv. Chem. Phys. 80, 1 (1991).

${ }^{12}$ R. Vasanthi, S. Ravichandran, and B. Bagchi, J. Chem. Phys. 114, 7989 (2001).

${ }^{13}$ J. G. Gay and B. J. Berne, J. Chem. Phys. 74, 3316 (1981).

${ }^{14}$ E. de Miguel, L. F. Rull, M. K. Chalam, K. E. Gubbins, and F. van Swol, Mol. Phys. 72, 593 (1991).

${ }^{15}$ The potential has been developed and tested in collaboration with Dr. S. Ravichandran, UMDNJ, Newark, New Jersey 07103 and Dr. A. Perera of LPTL, Univ. P. et M. Curie, Jussieu, Paris 75252 (private communication). This potential was used in Ref. 12 and was shown to give consistent results.

${ }^{16}$ H. Shimizu, J. Chem. Phys. 37, 765 (1962).

${ }^{17}$ S. Ravichandran and B. Bagchi, J. Chem. Phys. 111, 7505 (1999). 